\title{
Correction: A route out of resistance
}

Nature Microbiology 1, 16089 (2016); published 26 May 2016; corrected 13 June 2016

The original version of this Editorial incorrectly credited the need for new diagnostics to identify resistance profiles to the Pew roadmap, which makes no such suggestion. We apologize for any confusion this may have caused; all versions of the Editorial have been modified to rectify this error. 